\title{
Habitar digital, arquitectura sensible al hombre
}

\author{
Inhabiting digital architecture sensitive to man
}

<RESUMEN>

Arquitectura interactiva es un concepto que cada vez más se extiende desde el área de la informática al diseño espacial. Este artículo presenta tres casos en los cuales es posible evidenciar, como a diferentes escalas la incorporación de tecnologías computacionales en el espacio están generando nuevas plataformas digitales y de sociabilización.

$<A B S T R A C T>$

Interactive architecture is a concept that is increasingly trespassing the boundaries of information technology towards architectural design. This paper presents three cases that expose, at different scales, how Embedded Computer Systems have been introduced into our built environment. generating new digital platforms and socializing experiences.

$<$ PALABRAS CLAVE >

ARQUITECTURA ADAPTABLE / PANTALLAS URBANAS / INSTALACIONES INTERACTIVAS / INTERFACES HUMANOCOMPUTADORES (HCI) / INTERACCIÓN SOCIAL

KKEY WORDS>

ADAPTIVE ARCHITECTURE / URBAN SCREENS / INTERACT IVE INST ALLATIONS \& HUMAN-COMPUTER INTERFACE (HCI) / SOCIAL INTERACTION

\section{Introducción}

Tradicionalmente, hemos pensado en arquitectura como espacios conformados por elementos estáticos, ya sean losas, muros, vanos o cubiertas. Como resultado: edificios que para acomodar cambios deben ser demolidos o reconstruidos. A su vez, hemos entendido tecnología computacional como computadores sobre nuestros escritorios, los cuales a partir de las últimas décadas han comenzado a miniaturizarse y camuflarse en nuestro entorno físico. Microchips integrados a equipos portables como, laptops, teléfonos móviles, juegos de consolas, Mp3 players, GPS, entre otros, han permitido extender el uso de computadores más allá de las actividades solamente laborales e integrarlos a nuestras vidas cotidianas, dando pie a la llamada Revolución de la Información ${ }^{1}$.
Miniaturización, portabilidad, conectividad «wireless» e información digital disponible en cualquier lugar y momento gracias a Internet, son factores que definitivamente están transformando nuestros patrones de comportamiento social, nuestra relación con el cuerpo y con el espacio físico que nos rodea. Es así, como la Arquitectura enfrenta un nuevo desafío de participar en este cambio social, interactivamente respondiendo a nuevas necesidades del usuario del siglo xxI, e invitando a una integración de tecnologías digitales en nuestros entornos físicos y contextos sociales.

Generaciones de arquitectos e ingenieros han lidiado con el tema, de cómo lograr hacer edificios que sean capaces de adaptarse a sí mismos, ya sea para satisfacer dinámicamente nuevas necesidades programáticas de

\footnotetext{
Arquitecto de la Universidad de Chile con estudios de pregrado en L'Ecole d'Architecture de Burdeos, Francia. A desarrollado dos programas de magíster: MSc Adaptive Architecture and Computation en The Bartlett School, University College London y MA Computer Imaging in Architecture, University of Westminster, ambos en Londres (Inglaterra). Ha sido becada por la comisión Europea Alban, por la Universidad de Westminster y por la Universidad Diego Portales de Chile. Ha sido ayudante y profesor de taller de las escuelas de Arquitectura de la Universidad de Chile y Universidad Diego Portales. Ha sido invitada a dar ponencias y realizar workshops en la Universidad de Londres (Inglaterra). Su trabajo ha sido expuesto y publicado en diversos medios, galerias y conferecias. Ha trabajado profesionalmente en Santiago, Burdeos y Londres.

Castells, M. The information age: Economy, society and culture. Malden, MA: Blackwell, 1996.
} 

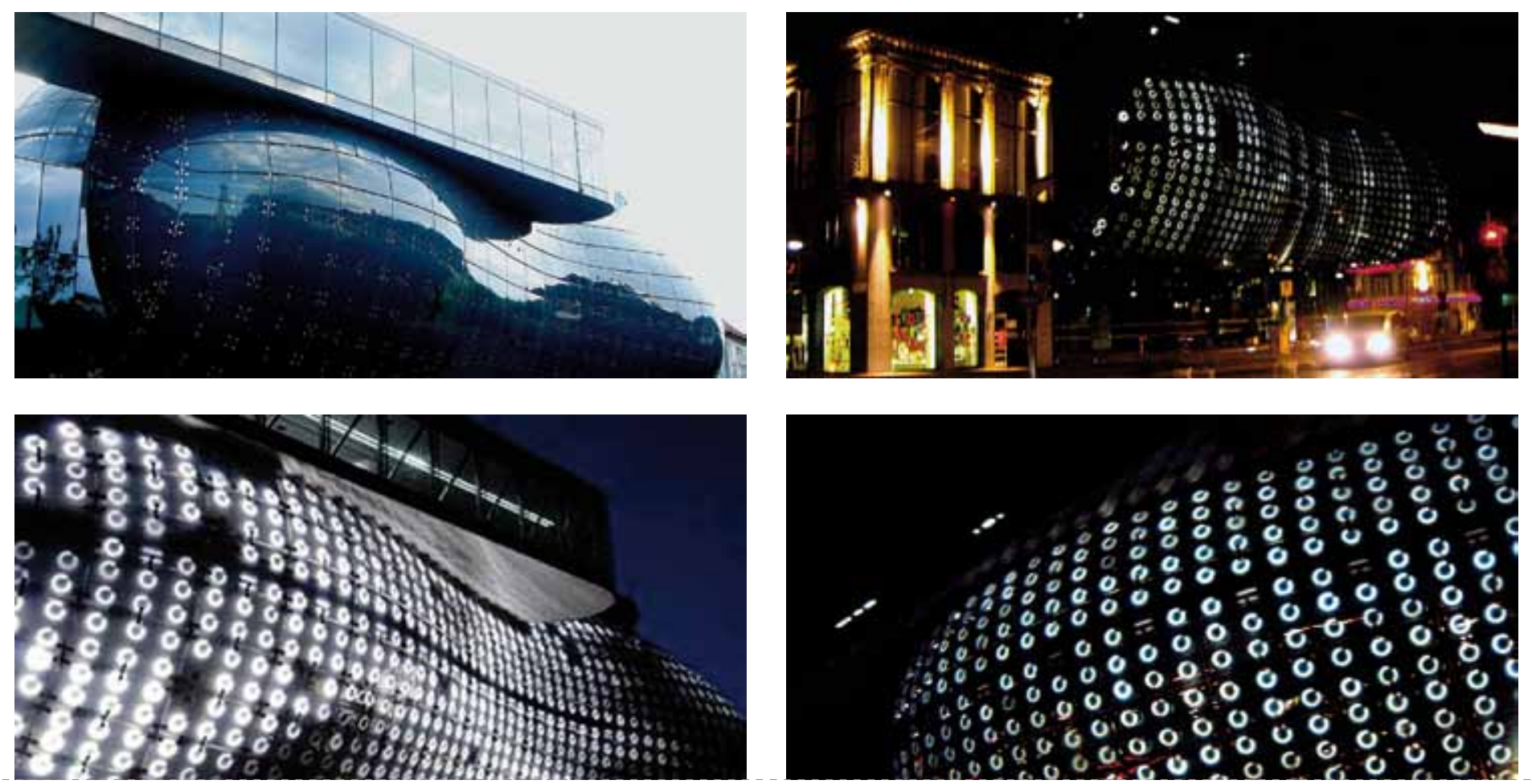

sus usuarios, o bien, para adaptarse sustentablemente a las variaciones de su contexto urbano y natural. Aunque el desarrollo de lo que podría llamarse una Arquitectura Robótica aún se ve un tanto lejano, los avances tecnológicos y la mayor accesibilidad económica a estas nuevas tecnologías, han permitido erigir proyectos que comienzan poco a poco a diluir las fronteras entre informática, arquitectura y arte.

El presente artículo expone tres casos en los cuales se yuxtaponen arquitectura y tecnologías digitales, que confrontados a sus escenarios sociales logran plasmar los nuevos lenguajes y contenidos mediáticos incorporados progresivamente en las nuevas sociedades contemporáneas. El primer caso corresponde a Kunsthaus Graz, un centro de exhibición de arte, emplazado en la ciudad de Graz (Austria), el cual introduce en su fachada una pantalla a escala urbana, la cual proyecta contenidos mediáticos generados por artistas.

El segundo proyecto, Son-O-House (Holanda), da cuenta de la introducción de tecnologías computacionales dentro del espacio físico del pabellón. El cual, por medio de sensores, logra captar los movimientos de sus visitantes en él, movimientos que activamente influyen y modifican los sonidos musicales generados en el pabellón.
El tercer proyecto, LEDs Urban Carpet (Inglaterra), es un proyecto desarrollado por el autor con el propósito de obtener el grado de máster en The Bartlett School, UCL. Este proyecto es una instalación interactiva, la cual logra captar el movimiento de los usuarios sobre una alfombra de luces. Esta alfombra responde dinámica y lumínicamente a los movimientos de los peatones sobre ésta.

En conjunto, pero a diferentes escalas, los tres casos expuestos pretenden exponer un nuevo marco teórico y práctico, dilucidando modos en el cual la introducción de tecnologías digitales y elementos dinámicos dentro del paisaje urbano, pueden permitir enriquecer un diálogo entre arquitectura y entorno, y entre arquitectura y habitante. En estos casos arquitectos y artistas se convierten en diseñadores de interacciones y coreografías conjugando armónicamente acciones/ reacciones de los edificios con los estímulos producidos por sus usuarios, donde el espacio físico interactúa con sus usuarios por medio de proyecciones en las murallas, equipamientos inteligentes o sensores, elevando al visitante a adoptar un rol activo y generativo, dentro de una arquitectura sensible al hombre.

\section{Pantallas urbanas}

La incorporación de pantallas gigantes en las fachadas de los edificios ha sido un recurso altamente explotado desde el área comercial y publicitaria hace ya varias décadas. Clásicos casos como $5^{\text {th }}$ Avenue en Nueva York o Picadilly Circus en Londres, son reflejos de una era fuertemente influenciada por el mensaje visual y por el constante intercambio de información.

A pesar de que estos edificios cubiertos por pantallas audiovisuales logran incorporarse rápidamente a su contexto urbano en constante ebullición, dinámico y vanguardista, niegan por completo la clásica relación arquitectónica entre interior y exterior de un edificio, entre programa y fachada.

Kunsthaus Graz (2003), desarrollado por los arquitectos Peter Cook y Colin Fournier, a diferencia de los casos anteriores, es un edificio que incluye en su arquitectura una fachada performativa pero sin propósito comercial, sino más bien -elaborada como tablero de experimentación- está dedicada a mostrar producciones artísticas relacionadas con su uso interior.

El edificio es un museo internacional para exhibiciones de arte contemporáneo y nuevos medios. A diferencia de los museos 
tradicionales, éste no posee una colección privada ni permanente, por lo tanto los contenidos a exponer dentro del museo varían constantemente. Es así, como de acuerdo a los arquitectos ${ }^{2}$ el proyecto fue concebido como una "caja negra», donde cada exhibición transformaría y adoptaría el espacio de acuerdo a sus específicas necesidades espaciales.

Para los arquitectos, el encargo de que el edificio debía ser lo suficientemente flexible como para albergar diferentes clases de exhibiciones, ponía al proyecto ante dos posibilidades: o bien, hacer de él una estructura distintiva e iconográfica -así como el Museo de Guggenheim en Bilbao, Españao adoptar una postura más tradicional de entender el museo como un escenario en donde el arte expuesto sea el que prevalezca por sobre el edificio. En este caso los arquitectos se inclinaron por la primera opción, generando un edificio que se distingue por sus formas orgánicas y biomórficas, haciéndolo parecer un extraño animal.

Según la gente de Graz, Kunsthaus es considerado como un amistoso alien que constantemente trata de comunicarse y entregar mensajes por medio de su pantalla urbana monocrómica de baja resolución. Esta pantalla cuenta con una matriz de 930 luces fluorescentes en forma de anillos de $40 \mathrm{~cm}$ de diámetro, las cuales cubren un área de su fachada de aproximadamente 20 metros de alto por 40 metros de largo.

Cada una de sus luces actúa como un pixel, en el cual su intensidad de luz puede ser variada y controlada a una rapidez de 18 frames por minuto. Esto permite que la fachada proyecte patrones a toda su extensión lo cuales -al no ofrecer una alta definición- pueden ser mejor observados desde una considerable distancia. Desde una corta distancia, lo que llama la atención es la presencia de acción cuando la fachada pasa de estar opaca a encenderse, no se puede ver una imagen clara, sino más bien el peatón debe intentar interpretar y quizás poner de su propia imaginación para reconstruir un mensaje.

Esta matriz de contenido multimedia, parece estar tatuada en el edificio, integrada en la piel de este animal, es cómplice del programa que el edificio alberga. Compartiendo la misma escala, arquitectura e instalación mediática, ambas logran emerger un nuevo resultado estético. Kunsthaus, ya sea por su compleja forma curvilínea o por su llamativa fachada performativa, logra generar tal atracción dentro de la ciudad de Graz, que ha logrado a escala urbana regenerar parte del lado Oeste de la ciudad, el cual fue hasta ahora relativamente desaventajado en relación al resto de la ciudad.

Este edificio logra comunicar de una forma textual la importancia del mensaje. Sobre todo de un mensaje mediático dominado por el valor de lo visual, del contenido estético y cambiante.

\section{Plataformas sensibles al hombre}

Lograr hacer información digital tangible, ha sido la posición de MIT Media Laboratory desde que el concepto «tangible bits» ${ }^{3}$ fue desarrollado por Hiroshi Ishii en el año 1998. Bajo este concepto, el grupo ha explorado modos que permiten al hombre acercarse cada vez más a manipular físicamente información digital, por medio de la integración a objetos comunes de cada día, e introducirla en nuestros espacios diarios de trabajo.

De acuerdo con Hiroshi Ishii, la interacción entre el usuario y el ciberespacio ha sido confinada a tradicionales interfaces computacionales -como computadores de escritorios o consolas de juegos- los cuales están separados de nuestro espacio físico cotidiano en donde vivimos y nos relacionamos con otros. Según el autor, por medio de «tangible bits», es posible ligar lo que actualmente se encuentra separado, la relación entre ciberespacio y nuestros entornos construidos.

Por otra parte, Mark Weiser ${ }^{4}$ (1995) y su concepto «ubiquitous computing» remarcan la idea de que en un mundo cada vez más computarizado, las tecnologías digitales comienzan a introducirse y desaparecer en nuestros escenarios físicos, pero de una forma casi imperceptible, encarnando objetos cotidianos con los cuales logramos relacionarnos natural y espontáneamente. Weiser propone, exportar las tradicionales interfaces computacionales e integrarlas a elementos arquitectónicos y urbanos, ya que a través de éstos se pueden generar nuevas formas de experimentar la ciudad, de construir comunidad y barrios, provocando quizás nuevas formas de interacción social y de encuentros.

Hoy en día la introducción de tecnología digital a elementos tangibles ha cambiado el tradicional concepto de arquitectura, invitándonos a considerar nuestros entornos físicos como superficies sensibles a los movimientos humanos, sonidos, olores, temperatura o quizás ondas de radio o Wi-fi. La arquitectura interactiva, va en busca de proporcionar una plataforma donde animar las experiencias entre el usuario en relación al espacio que lo rodea.

Para lograr esto, este medio requiere «inputs» o estímulos por parte del entorno o el usuario, los cuales son leídos y analizados por medio de sensores introducidos al espacio. Los sensores son la pieza clave de cualquier arquitectura interactiva, ya que permiten construir el puente entre personas y computadores. Los sensores pueden medir cuantitativamente: presión, variaciones lumínicas, sonidos, calor, electromagnetismo, entre otros. Estos estímulos deben ser leídos por un microchip o microcomputador, quienes a su vez activan sistemas que responden físicamente a los estímulos generados, "outputs».

El siguiente caso está localizado en una larga área industrial, al costado de una autopista en la zona de Ekkersrijt, Holanda. Son-OHouse $^{5}$ (2004) es un pabellón público donde los visitantes se pueden sentar a almorzar, a reunirse, encontrarse o simplemente disfrutar del medio ambiente. La estructura tiene un propósito arquitectónico, como también el de albergar una instalación acústica, que permite a sus visitantes, no solamente escuchar una música ambiental, sino participar activamente de su composición. El proyecto es concebido como una especie de instrumento o estudio musical a escala urbana ${ }^{6}$

\footnotetext{
Cook, P., Fournier, C. A friendly alien: Ein Kunsthaus für Graz. Kunsthaus Graz AG (Hg): Dieter Bogner, 2004. Factors in Computing Systems. Los Angeles, CA, EE.UU., 1998.

Weiser, M. «The computer for the 21 $1^{\text {st }}$ century». Scientific American 1991.

En: http://www.noxarch.com/flash_content/flash_content.htm

6 Bullivant, L. 4dspace: Interactive Architecture. Architectural Design Magazine. Chichester: Wiley-Academy, 2005.
}

3 Ishii, H., Wisneski, C., Brave, S., Dahley, A., Gorbet, M., Ullmer, B., Yarin., P. «AmbientROOM: Integrating ambient media with architectural space». Conference of Human 

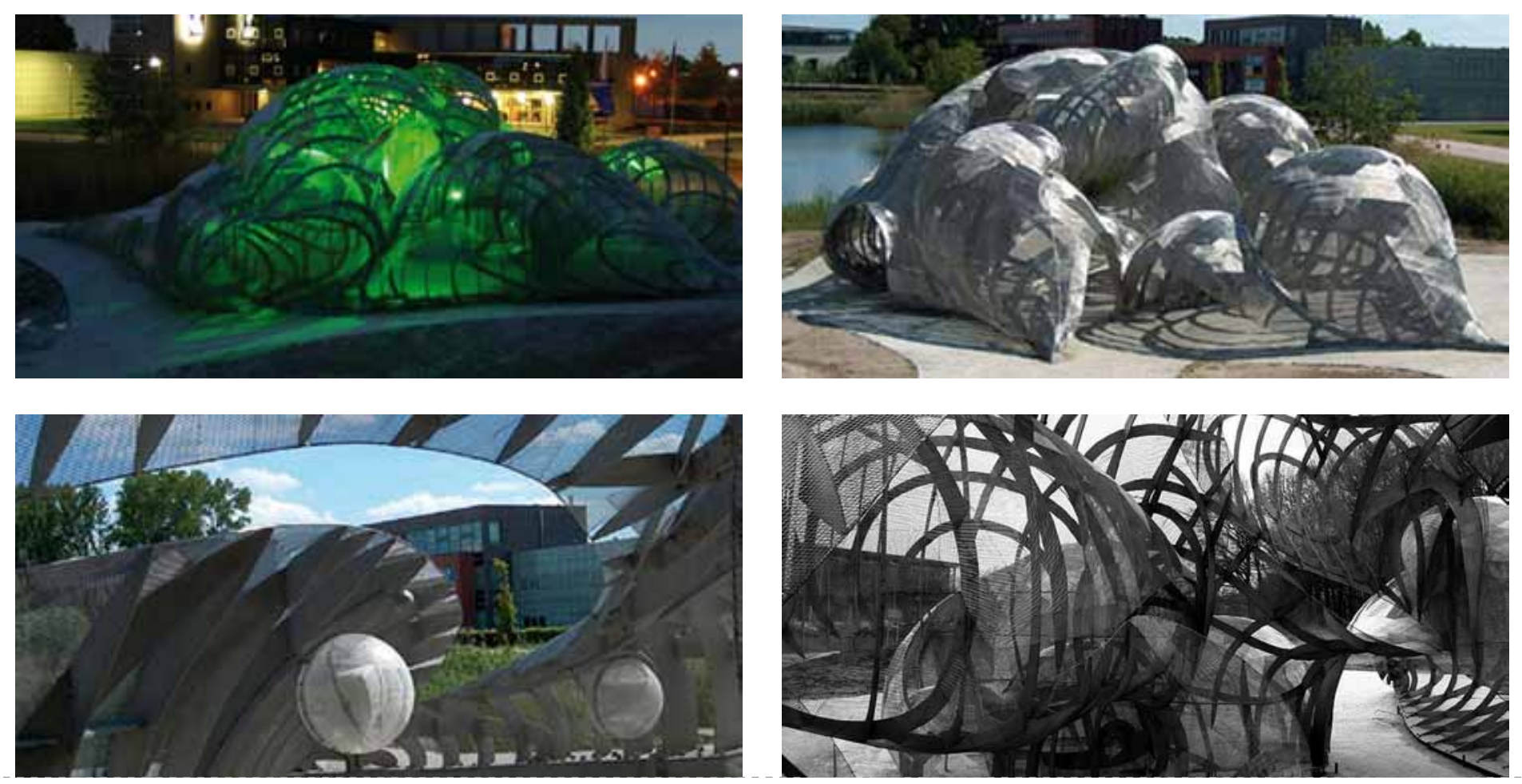

Su forma metálica curvilínea, fue en sí generada siguiendo el trazo de movimientos humanos. Proyectado por Lars SpuybroekNox, en conjunto con Edwin van der Heide ${ }^{7}$ (compositor); el pabellón está continuamente produciendo nuevos patrones musicales de acuerdo a los movimientos de sus visitantes dentro de él. Estos movimientos son detectados por 23 sensores de sonido localizados estratégicamente dentro del espacio. De acuerdo a sus movimientos, el usuario influye directamente en la línea de tiempo de la composición musical que se está emitiendo.

Dentro del pabellón se escucha un casi imperceptible sonido digital; una vez que el visitante logra evidenciarlo, comienza a darse cuenta que por medio de sus movimientos corporales logra modificar el sonido dinámicamente. La percepción del espacio varía continuamente sin fin, reconfigurándose de acuerdo a los movimientos de uno o más usuarios al interior de él. Este caso logra hacer que la tecnología digital sea más fácil de entender, y a su vez más cercana al cuerpo y sentidos del hombre.

\section{Instalaciones interactivas}

El concepto de medios digitales interactivos, ha sido relegado en su mayoría al uso de video juegos, de DVDs o al diseño de sitios de Internet. Sin embargo, el actual desafío de arquitectos, diseñadores y artistas ha sido el de extender este medio a escala humana, sensible a nuestro cuerpo y sentidos. Tomando la forma de instalaciones, arte público, o espacios de entretención, se ha comenzado a generar plataformas donde se conjugan arte, medios digitales e interacción social. Objetos que responden a la presencia del cuerpo o a los equipos electrónicos portables que llevamos con nosotros.

«Experience Design» ${ }^{8} 0$ «Diseño de Experiencias» es una proposición conceptual que pretende generar relaciones emocionales con individuos, quienes estimulados a un nivel sensorial logran interactuar entre ellos y con ambientes inteligentes. Este diseño está basado en el estudio y entendimiento de los usuarios y de su comportamiento en un contexto físico y social. Con el propósito de hacer visible lo que siempre parece invisible o dejado de lado, como: nuestros movimientos corporales, identidades, caras, poluciones, ruidos, etc. Intentan reescalar nuestros efectos sobre el espacio, elevando al usuario a un nuevo nivel de control e interacción con su entorno.

LED's Urban Carpet ${ }^{9}$, es una instalación interactiva diseñada para ser introducida en contextos urbanos y entornos sociales, con el propósito de estimular la interacción social entre familiares, amigos o desconocidos, y a su vez estudiar el impacto a nivel espacial y de integración social cuando se introducen nuevas tecnologías tangibles en ambientes públicos.

Consiste en una especie de alfombra plegable, capaz de ser transportada con la intención de ser introducida y testeada en diferentes situaciones urbanas. La instalación mide aproximadamente 280 x 180 cms, y está conformada por dos capas que en conjunto no alcanzan una altura mayor a $3 \mathrm{~cm}$. La primera capa consiste en una grilla de 18 sensores de presión, los cuales sienten el peso de los peatones y envían información sobre ubicación y recorridos de los usuarios sobre la alfombra, hacia un computador.

En: http://www.evdh.net/index.html

Bullivant, L. Responsive environments: Architecture, art and design. Londres: V\&A Publications, 2006.

Briones, C., Fatah, G., Schieck, A., Mottram, C. «A socializing Interactive Installation for the urban environments». IADIS Applied Computing 2007. 


\section{No 16}

de arquitectura

LEDs Urban [arpet (Inglaterra) [fotografías de Ava Fatah gen. Schieck].
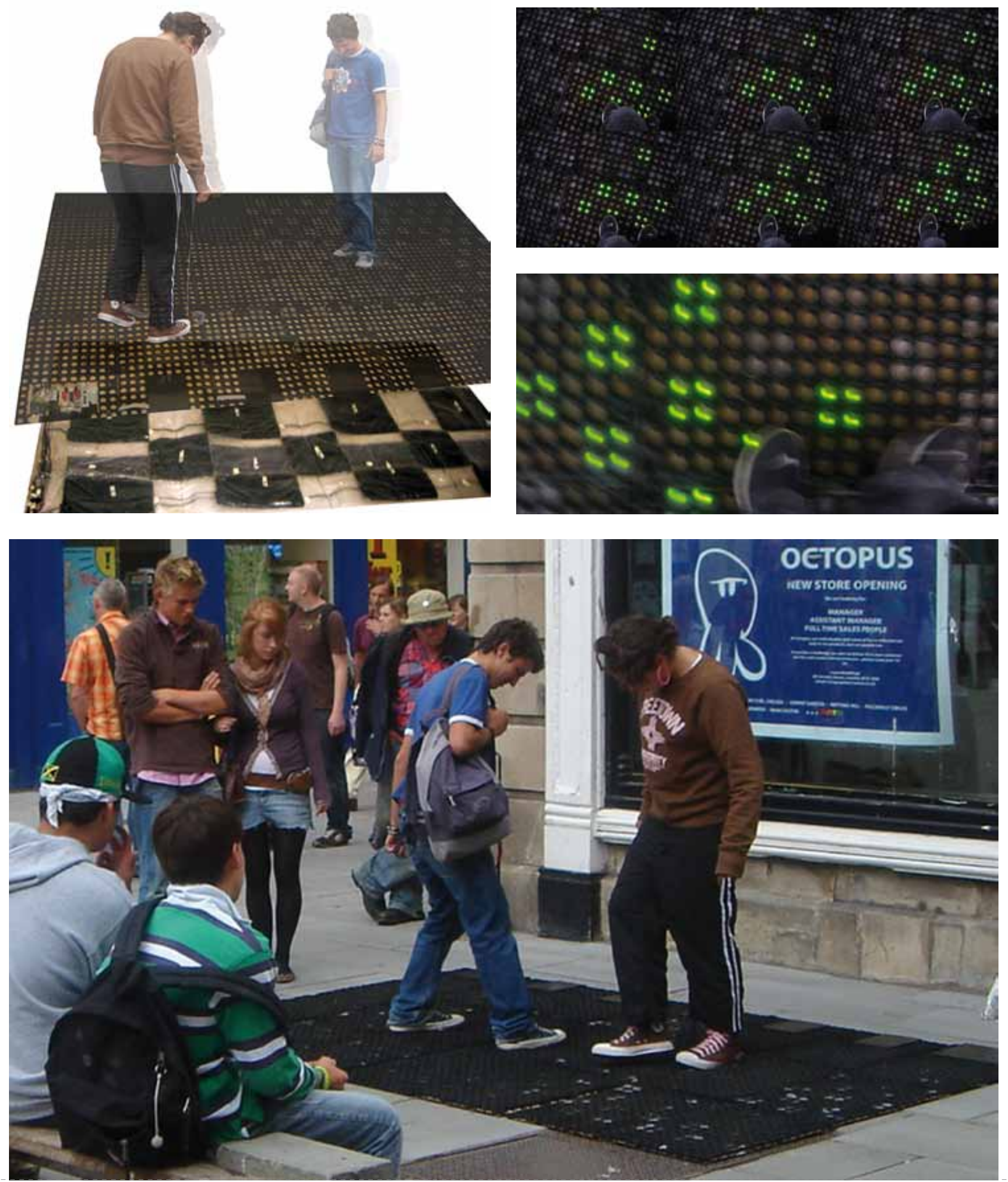
La segunda capa consiste en una alfombra de goma la cual alberga 840 modulos de LEDs (diodos emisores de luz). Esta capa reacciona de acuerdo a los movimientos de los usuarios, encendiendo y apagando luces. La instalación genera patrones lumínicos que simulan el vuelo de una bandada de aves que siguen y revolotean alrededor del peatón.

La interface en sí misma siempre se encuentra emitiendo patrones lumínicos, sin embargo, una vez que un participante camina sobre ella, el usuario tarda un tiempo en evidenciar que estos patrones comienzan a reaccionar a su paso por sobre la alfombra, ya que los patrones de luz no reaccionan directamente al recorrido del usuario, sino que son programados como una identidad inteligente en sí misma, la cual más que reaccionar al estímulo directo del usuario, tratan de imbuirlo a él en un constante juego.

La instalación, por medio de su contenido mediático, desea generar una atmósfera de entretención y juego espontáneo entre sus usuarios. Quienes de una forma sencilla y libre sienten la curiosidad de interactuar tanto con la interface inteligente como con la gente alrededor, ya sea por el hecho de compartir una experiencia o por hacer el juego aun más impredecible. Ya que a mayor cantidad de participantes interactuando al mismo tiempo con la instalación, más aleatorio es el comportamiento de las aves digitales.

El proyecto ha sido testeado en exhibiciones en The Bartlett School, UCL y en Architectural Week 2007 ambos en Londres, como también durante una semana en la patrimonial ciudad de Bath en Inglaterra. LED's Urban Carpet ha dado cuenta de cómo peatones se sienten atraídos por el uso de nuevas tecnologías más allá de sus contextos laborales, revelando también lo atractivo y muchas veces divertido que es para los participantes relacionarse con interfaces digitales por medio de su cuerpo y a través de sus propios estímulos.

Este proyecto también remarca el potencial que tienen los nuevos medios de generar plataformas de cohesión social y reconocimiento del espacio físico que nos rodea, y no solamente situaciones inmersistas aisladas donde la relación hombre-máquina prevalece por sobre las relaciones humanas.

\section{Habitar digital}

A través de los casos presentados anteriormente, es posible entrever que una nueva dimensión de las tecnologías digitales aplicadas al espacio arquitectónico está transformando nuestras identidades espaciales y nuestra forma de relacionarnos con el entorno.

El potencial que adquieren estas interfaces no solamente está relegado a un aspecto de entretención. También han comenzado a proporcionar soluciones para áreas educativas, comerciales, retail, museos, entre otras, reinventado nuestros espacios de trabajo, doméstico, goce u ocio, sin obviar la básica necesidad humana de un espacio físico en el cual subsistir y relacionarse con otros.

«Interactividad» puede ser entendida como la capacidad de la audiencia de participar y en un cierto grado controlar la obra de arte o representación. Hasta ahora, nuestras culturas han permitido muy poco control del usuario a modificar o influir la obra o el espacio ya diseñado previamente. Sin embargo, al usuario contemporáneo se le están abriendo cada vez más puertas a tener un acceso fácil, libre y democrático, a nuevas plataformas inteligentes capaces de reconfigurarse de acuerdo a necesidades o estímulos de quienes las habitan, lo cual sin duda, dará pie a nuevas formas espaciales y nuevas organizaciones sociales emergentes, en una era dominada por las redes de información y colaboración. 